Math. Model. Nat. Phenom.

Vol. 6, No. 6, 2011 pp. 260-277

DOI: $10.1051 / \mathrm{mmnp} / 20116614$

\title{
A Team Approach to Undergraduate Research in Biomathematics: Balance Control
}

\author{
J. Milton ${ }^{1 *}$, A. Radunskaya ${ }^{2}$, W. Ou ${ }^{3}$, and T. Ohira ${ }^{1 \dagger}$ \\ ${ }^{a}$ Joint Science Department of Claremont McKenna, Pitzer and Scripps Colleges \\ W. M. Keck Science Center, 925 N. Mills Ave., Claremont, CA 91711, USA \\ ${ }^{b}$ Department of Mathematics, Pomona College, 610 North College Ave. \\ Claremont, CA 91711 USA \\ ${ }^{c}$ Department of Mathematics, Scripps College, 1030 Columbia Ave., CA 91711, USA
}

\begin{abstract}
The question, how does an organism maintain balance? provides a unifying theme to introduce undergraduate students to the use of mathematics and modeling techniques in biological research. The availability of inexpensive high speed motion capture cameras makes it possible to collect the precise and reliable data that facilitates the development of relevant mathematical models. An in-house laboratory component ensures that students have the opportunity to directly compare prediction to observation and motivates the development of projects that push the boundaries of the subject. The projects, by their nature, readily lend themselves to the formation of inter-disciplinary student research teams. Thus students have the opportunity to learn skills essential for success in today's workplace including productive team work, critical thinking, problem solving, project management, and effective communication.
\end{abstract}

Key words: inverted pendulum, time-delay, noise, dimension reduction, pursuit, undergraduate, education

AMS subject classification: 97M60, 92B99, 34K20

\footnotetext{
*Corresponding author. E-mail: jmilton@jsd.claremont.edu

$\dagger$ Sony Computer Science Lab, Tokyo, Japan
} 


\section{Introduction}

Living organisms are continually in motion: birds fly, snakes slither, worms wiggle and people fidget. Although it would be anticipated that discussions of movement would dominate courses and textbooks in undergraduate bio-mathematics, in fact the topic is seldom mentioned (for notable exceptions see $[5,31,32,60]$ ). One explanation is the misconception that motion is a "solved problem". However, Newton's laws of motion do not describe how motions are controlled and shaped to optimally meet the needs of organisms.

Current approaches to the study of the movements of living organisms emphasize that the brain has a body [13]. This concept implies that movements are controlled by both passive and active mechanisms [16, 34]. Active control describes the contribution of forces that arise from muscle contractions determined by neural motor programs. Passive control describes the effects of forces related to the biomechanical properties of the body and the changing environment in which the movements occur. The problem of determining how much of the control of movement is active and how much is passive is made complex since this relative proportion can change depending on the immediate goals of the organism.

Mathematical modeling forms the foundation for a three-step strategy to investigate the nature of movement control. First, provided that the movement is not too complex, mathematical models based purely on classical mechanics, i.e., a model that ignores both the body and the brain of the organism, can be developed. Much discussed examples include problems related to the flight of a projectile (golf balls, basketballs, baseballs) [2, 64], racketball bouncing [16], stick balancing at the fingertip [9, 20, 36] and the searching patterns of organisms $[5,23,59]$. Second, comparisons between prediction and observations invariably show discrepancies. The development of affordable high speed motion capture technologies has greatly facilitated the recognition of these differences. Finally, and this is the fun part, we can use mathematics to identify the nature of the contributions made by the body and brain that account for the discrepancies.

Our impression is that science and engineering undergraduates are overwhelmingly attracted to problems related to movement. Their fascination stems from their interest in fitness and athletics, but also is strongly motivated by issues related to rehabilitation, aging, and more recently, to the restoration of movement by the use of neuro-robotic prosthetic devices (see, for example, [24]). It is self-evident that an undergraduate initiative which harnesses these student interests would provide a pathway to encourage them to use mathematics in biological research. However, problems related to the description of movement typically require skill sets beyond those attained by typical undergraduate science students. An exception concerns the study of balance and balance control. In Section 2. we describe our team-based research program in motion science. The success of our program is best illustrated through a description of three projects that our faculty-student teams are currently pursuing (Section 3.). The purpose of these descriptions is to emphasize the rich variety of interesting mathematical problems, many yet to be solved, which arise. Overall our efforts provide strong support for the fundamental truth of education, namely, a student motivated to learn will exceed all expectations of their educators. 


\section{Motion science program}

The motion science program at the Claremont Colleges has evolved over seven years. It was specifically designed to provide opportunities for students and faculty to work together as members of an inter-disciplinary teams [40]. The choice to focus on the area of motion science was deliberate and not based on the prior experiences of the authors. We were attracted to motion science because of 1 ) the interest of our students in problems related to movement and 2) the ease by which long time series collected with high spatial and temporal resolution can be collected using motion capture technology. The availability of such data greatly facilitates direct comparisons between experimental observations and models based on differential equations. The cost of motion capture systems have decreased dramatically in recent years. Although high speed, high precision commercial motion camera systems remain costly (e.g. Vicon, Opti-Trak, Qualisys), the recent trend has been towards the development of computer software packages that can readily use less expensive digital video cameras for motion capture (e.g. Innovision Systems).

\section{1. $\quad$ Team-based model}

Many different skills are required to conduct research in motion science. This observation suggests a team-based approach to answering questions about human balance and locomotion. Students were motivated to participate through their pre-existing interests in sports, the development of skill and expertise, biomechanics, the prevention of falling in the elderly, and the development of neuro-robotic prostheses for rehabilitation. Consequently we attracted students having a wide range of backgrounds including those who were clinicallyoriented (dance, human biology, martial arts, pre-med) and those who were computationallyoriented (computer science, mathematics, physics, engineering). We leveraged the diverse backgrounds of our students through the formation teams consisting of 2-3 students whose combined skill set spanned the requirements for each project [40]. All members of the research team participated in all aspects of the study at a level determined by their interests and abilities; however, typically mathematically-oriented students took the lead for the development of the model, the laboratory-based students the lead for experimental design and data analysis and the team worked together on comparisons between predictions and experimental observations. Faculty are active members of the team. They worked side by side with the students, often participated in the experiments, and provided the hands-on expertise needed to extend investigations into directions that lie outside the scope of an undergraduate science education. The active involvement of faculty in student research projects is necessary in the setting of a liberal arts college since graduate students and postdoctoral fellows are not available. Moreover the formation of faculty-student inter-disciplinary teams provides educational opportunities to teach students the skills that are most required in the workplace, namely, productive team work, critical thinking, problem solving, project management, and effective communication [40].

Hands-on research experiences are provided through 8-10 week summer research projects, 
one semester independent study projects and two-semester senior thesis research projects. Typically it takes 2-3 consecutive team projects, i.e., 2-3 years, to bring the research to a point of publication in a peer-reviewed journal. Continuity is maintained by the faculty, student lab books, and the senior thesis prepared by the students to satisfy their graduation requirements. It should not be surprising that student team members develop a very strong sense of ownership for the project that they work on. Indeed, graduated students typically return the following semester to help train students on needed research skills and to share experiences. Student contributions are recognized in the author list of resulting publications (see, for example, [36, 38, 39, 41, 42]).

\subsection{Student preparation}

To date 43 students have participated in the motion science program. All of our students have strong interests in motion science. In addition to scholar athletes, dancers and martial artists, approximately $40 \%$ had hands-on experience working in physical therapy settings including the training room. Of the 13 team members who had previous training in differential equations, 7 were mathematics majors and 6 were neuroscience majors. Exposure to delay differential equations is provided through a specially designed course in introductory bio-mathematics (click on COURSES at rebmi.jsd.claremont.edu).

The principal quantitative tool used by all student participants is open source computing. Freely downloadable Python software packages are available to digitize data using standard A/D boards (PyUniversalLibrary [50]), to enable real time collection and analysis of uncompressed digital images from a variety of sources (motmot [58]), and to generate a wide range of visual stimuli for laboratory research (VisionEgg [57]). Of course many of the software packages for the analysis of differential equations are also freely down loadable from the Internet, for example, XPPAUT [17], PyDelay [49], and there is usually an open source version of essentially every commercial software package used by bio-mathematicians for numerical analysis (OCTAVE [44], SciPy [52]) and symbolic analysis (SAGE [51]). As these resources become more and more accessible it becomes possible to teach differential equations in a laboratory setting. In our experiences, the "light goes on" when a student realizes that the solution of a differential equation predicts the movements measured using a motion capture system and that this whole exercise was performed on their own laptop. Even students with little background in mathematics were able to develop programming skills sufficient to collect and analyze data. The advantage on our emphasis on computing skills for all was that the computer monitor became the focal point of team discussions, particularly when model was compared to observation.

\subsection{Faculty involvement}

During the first four years the goal was to develop a strong experimental program in motion science with a focus on balance control and falling in the elderly. This effort was facilitated by the fact that one of the authors (JM) is both experimentalist and modeler. Subsequently, 
faculty from mathematics and biology were attracted to the program because of the nature of the questions being asked and the possibility of using motion capture cameras to answer questions applicable to their own research. Thus, for example, AR participates through her interest in nonlinear dynamics and scale-free systems, TO because of his interest in delayed stochastic processes, and WO because of his interest in the development of dimension reduction techniques.

\subsection{Program evaluation}

The focus of our program is on team performance and the deliverable is a publication in the form of a senior theses and/or a peer-reviewed publication. In this way the team projects take on a very real world flavor. To date there have been 36 senior thesis and five peerreviewed publications [36, 38, 39, 41, 42]. Of the graduated students, 7 are pursuing careers related to computational science and 16 are pursuing clinical careers related to movement science (doctor of physical therapy, oriental medicine, orthopedics, and podiatry). However, by far the most compelling and exciting aspect of our program has been the ability of the student-faculty teams to generate novel insights into human balance control. Thus in the next section we describe three projects so that the reader can appreciate what motivated undergraduate students can produce when given the opportunity.

\section{Research projects}

The stabilization of an inverted pendulum plays a central role in discussions of balance control [62] and locomotion [25]. The dynamics of a frictionless inverted pendulum (Figure 1) are described by the differential equation $[4,48]$

$$
\ddot{\theta}-\frac{3 g}{2 \ell} \sin \theta=0
$$

where $\theta$ is the vertical displacement angle $(\theta=0$ is the inverted, upright position), $\ell$ is the length of the stick and $g$ is the acceleration due to gravity. The upright position is an unstable saddle point and hence even the slightest deviation from vertical causes the pendulum to fall over. The question is, How is the inverted pendulum stabilized in the upright position? attracts the interest of biologists given the impending epidemic of falling the elderly. In other words if we write (3.1) as

$$
\ddot{\theta}-\frac{3 g}{2 \ell} \sin \theta=F_{\text {control }}(t-\tau)
$$

then what choice of the feedback, $F$, best explains the experimental observations? In (3.2) we have written $F_{\text {control }}(t-\tau)$ to emphasize that neural feedback mechanisms for balance control are time-delayed where $\tau$ is the time delay [12, 27, 37, 55].

The fundamental nature of the control problem can be appreciated by regarding the relative movements of variables related to the center of mass (COM) as the controlled variable 


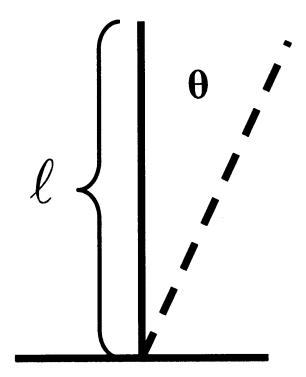

Figure 1: An inverted pendulum of length $\ell$ with uniformly distributed mass, $m$, and vertical displacement angle, $\theta$.

(for real stick balancing the position of the the tip of the stick or its mid-point) and variables related to the center of pressure (COP) as the controller [37, 62] (for stick balancing the position of fingertip). Viewed from this perspective the movements of the balanced stick are controlled by the relative positions of COM and COP: in 1-D as the COP moves to the right of the $\mathrm{COM}$, the $\mathrm{COM}$ moves left, and vice versa. Figure 2 demonstrates that in a variety of human balance tasks there is an oscillatory relationship between COM and COP. Motion capture studies have pointed to the complexity of balance control. In particular, the corrective movements for both stick balancing [9] and human postural sway [3, 6, 29, 30] are made intermittently. In the case of stick balancing at the fingertip $[9,10,14]$ a variety of power law behaviors have been identified, including intermittency and Lévy flights. Recently emphasized extensions to (3.2) include the introduction of a sensory "dead zone" to account for the fact that very small changes in $\theta$ cannot be detected by the nervous system, the inclusion of terms related to $\dot{\theta}$ and $\ddot{\theta}$ into $F[28]$, the inclusion of two time delays into $F$ [8], the assumption that $F$ is a discontinuous function of $\theta[18,36]$, the possibility that $F$ reflects an element of predictive control [33] and the possibility that the presence of power laws reflect, at least in part, the presence of an underlying optimal control mechanism $[19,46]$. The fact that $F$ is not yet determined served as an incentive for the student-faculty research teams: they could be the ones who first figure it out!

\subsection{Balancing with vibration: "Drift and act"}

It is well known that an inverted pendulum can be stabilized by vibrating the pivot point in a vertical direction using frequencies, $f$, that exceed

$$
f>\frac{\sqrt{2 g \ell}}{2 \pi a}
$$

where $a$ is the peak-to-peak amplitude $[1,48]$. The explanation for this phenomenon can be understood from the stability analysis of Mathieu's equation

$$
\ddot{\theta}+(\alpha+\beta \cos 2 \pi f) \theta=0
$$



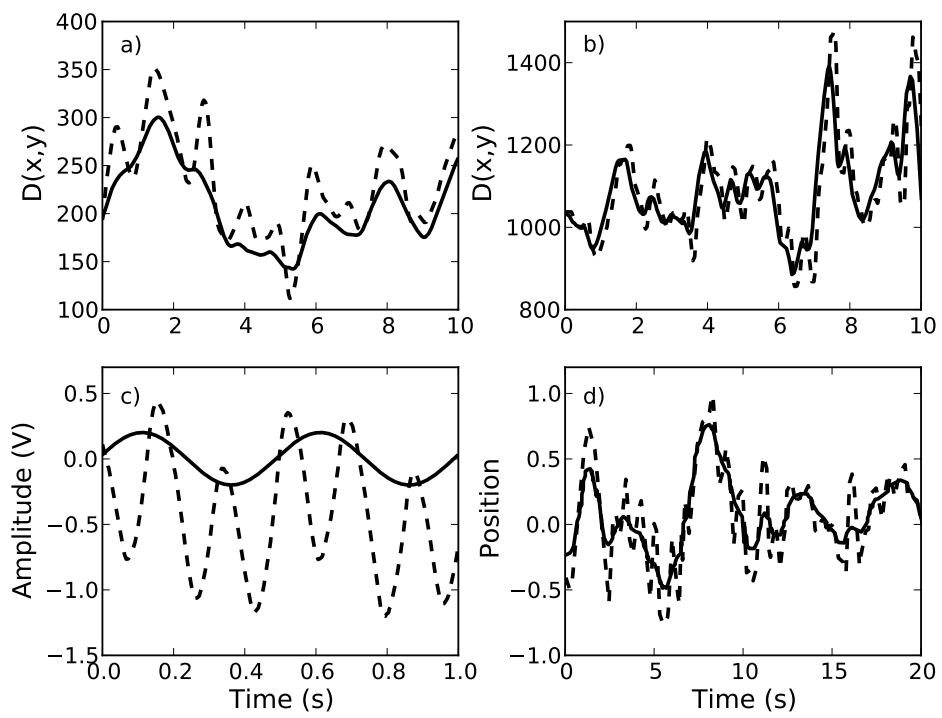

Figure 2: Comparison of the time course of a variable related to the center of mass (solid line) to a variable related to center of pressure (dashed line) for four human balance tasks: a) real stick balancing at the fingertip, b) virtual stick balancing on a computer screen (see Section 3.2.), c) mechanical stick balancing controlled by a time delayed feedback controller [37], and d) human postural sway [62]. $D(x(t), y(t))=\sqrt{x^{2}(t)+y^{2}(t)}$ is the length of the position vector measured at time $t$ from a common reference point [36], supplied, respectively, by the Qualisys motion capture system and the computer program. In c) the amplitude is measured as the voltage output of the potentiometers used to measure the angular displacement of the stick and the position of the cart. In $d$ ) the position is given as the displacement of the center of mass and the center of pressure from a centrally located reference point.

where $\alpha, \beta$ are constants. By comparing equations (3.1) and (3.3) we see that the effects of vibration enter through a parameter and hence this effect is an example of stabilization due to parametric excitation. Often overlooked is the fact that a necessary condition for stabilization is that the downward acceleration of the pendulum must at some point exceed that of gravity [48]. In other words the pendulum must be physically attached to the pivot point.

The student team (project onset 2007 involving 6 students to date: one dance-neuroscience major, one human biology major, one mathematics major, and three neuroscience majors) asked, Does vertical vibration of the fingertip have an effect on stick balancing even though the stick is not physically attached? Surprisingly the answer is yes when the upright position is controlled by time-delayed feedback [22, 36, 39]! Figures 3a and c show that introducing vertical vibration at the fingertip using a whole-body vibrator has a stabilizing effect on stick balancing, namely, the distribution of the fluctuations in the position of the fingertip narrows. Moreover, the average stick balancing time determined from multiple trials (typi- 

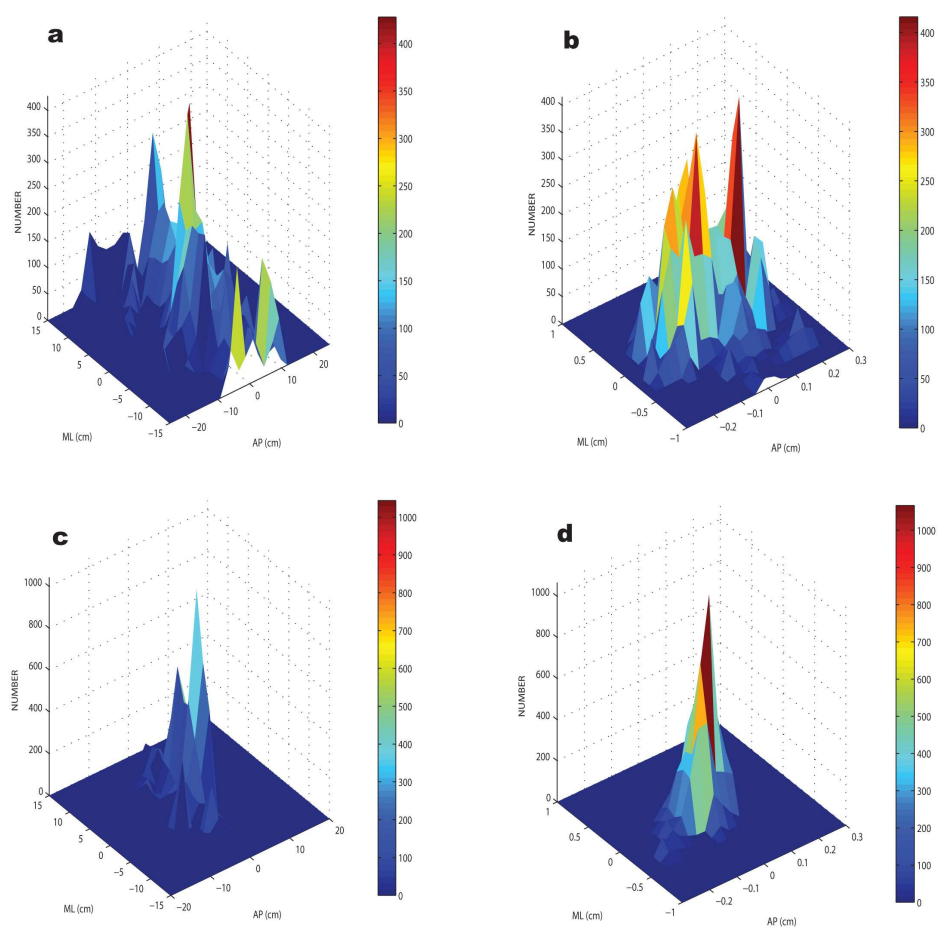

Figure 3: Effects of vibration on two human balancing tasks. The left column compares the effect of c) whole body vibration to a) no vibration on the medial-lateral ( $x$-axis) and anterior-posterior ( $y$-axis) position of the fingertip tip during stick balancing [36]. The right column compares the effect of d) low amplitude vibration of bilateral Achilles tendons to b) no vibration on the position of the center of pressure measured during quiet standing using a force platform [39]. Here the $x$-axis is the medial-lateral component of the COP and the $y$-axis is the anterior-posterior component of the COP.

cally $\geq 25$ ) is longer with vibration [36]. A sharpening in the distribution of the fluctuations in the COP is also seen for postural sway during quiet standing when the Achilles' tendons are subjected to low frequency, low amplitude periodic vibration (Figures $3 \mathrm{~b}$ and $\mathrm{d}$ ). These observations support the concept that human balance control is maintained by a simple "drift and act" mechanism [21, 35, 36, 37, 38, 53]. This mechanism proposes that the basin of attraction for the stabilized upright position is small enough so that escapes ("falls") are possible [54, 63]. Inside the basin of attraction trajectories "drift"; and corrective actions are taken only when trajectories leave the basin of attraction. Consequently any strategy that decreases the amplitude of the fluctuations in $\theta$ will have a stabilizing effect since they decrease the probability that the trajectory escapes the basin of attraction.

The main features of these observations can be qualitatively captured by a simple model which incorporates an unstable equilibrium point, a time-delayed switch-type controller and 


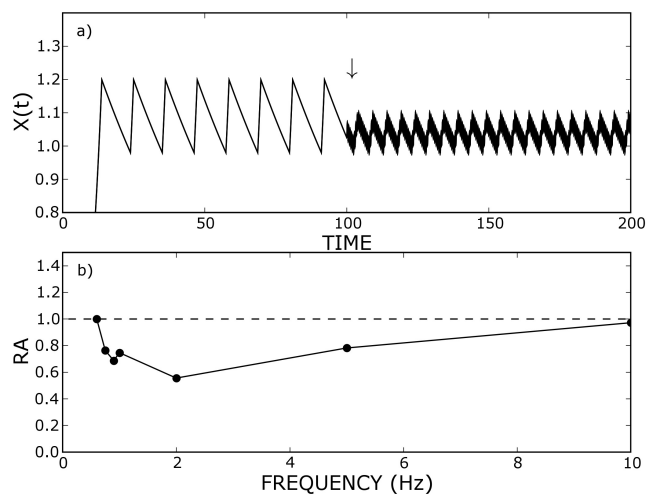

Figure 4: a) Dynamics of (3.4) for $a=0.18, b=-0.02$, and $k=0.3$. The periodic excitation is turned on at $t=100(\downarrow)$. b) The relative amplitude (RA) is the maximum amplitude in the presence of parametric excitation divided by the maximum amplitude in its absence. For $f<0.6$, high amplitude waveforms arise (i.e. $R A>1$ ) (not shown). Dashed line shows $R A=1$.

parametric periodic excitation [36, 39]

$$
\frac{d x}{d t}=F(x(t-\tau)) x(t)+k x(t) \sin 2 \pi f t
$$

where $k$ is a constant, $f$ is the frequency, $\tau$ is the time delay, and

$$
F(x(t-\tau)) \equiv \begin{cases}a & \text { if } x(t-\tau) \leq \theta \\ b & \text { otherwise }\end{cases}
$$

where $a>0>b$ and $\theta>0$. Equation (3.4) describes a "drift and act" controller: corrective actions ('act') are taken only when $x(t-\tau)>\theta$. Numerical simulations indicate that there is a range of parameters (e.g. Figure 4) for which a stable limit cycle exists when $k=0$ and when $k \neq 0$. In particular there is a decrease in the amplitude of the solution (greatest at $f=2$ ) and a decrease in the mean value of $x$. The thicker line in Figure 4a arises because the waveform becomes more complex in the presence of periodic forcing.

\subsection{Balance in virtual environments}

The student team (project onset 2008 involving 5 students to date: one human biology major, three neuroscience majors, and one mathematics major) asked whether the development of virtual balancing tasks that involve the interplay between a human and a computer could be used to investigate the nature of the neural control mechanisms [7, 11, 33, 46, 42]. The availability of open source software packages such as VisionEgg [57], a high level interface between Python and OpenGL, makes it possible for students to create a virtual stick balancing task. In this visuomotor tracking task the movements of the computer mouse are 

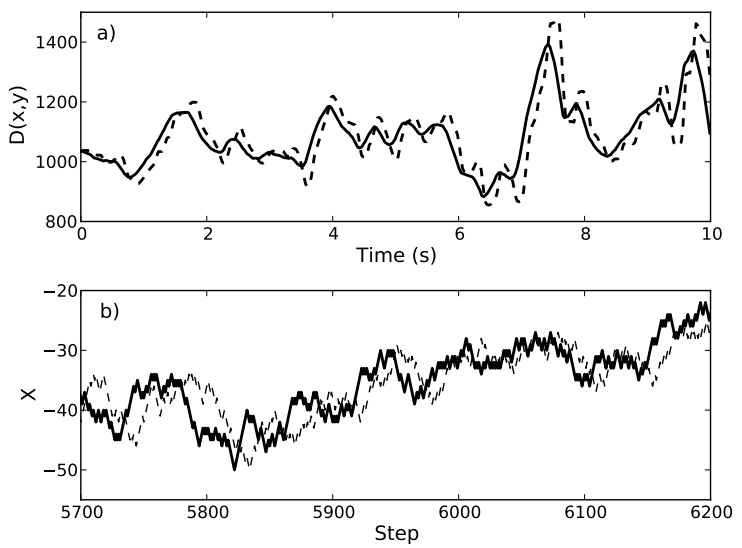

Figure 5: a) Position of the mouse (dotted line) versus the target (solid line) for a skilled subject. b) A single realization of the delayed pursuit task described by (3.7) for $\tau \sim 0.2 \mathrm{~s}$.

used to keep a target on the computer screen whose motions are governed by a parabolic potential centered at the mouse position $[7,11,46,42]$. The advantage of this paradigm is that key parameters can be manipulated experimentally.

Figure 5a shows that the relative movements of mouse and target for a skilled virtual stick balancer (mean survival time for 25 trials $\sim 1-2 \mathrm{~min}$ ) have the characteristics of pursuit and escape. Initially the movements of the fingertip pursue those of the stick's tip (hence we refer to this phase as pursuit); however, at some point the excursion of the position of the fingertip sufficiently passes that of the stick's tip. This causes the movement of the stick's tip to change direction (hence we refer to this phase as escape). After a lag, the movements of the fingertip again pursue those of the stick's tip. Consequently, for skilled subjects there is an oscillatory appearance of recurring, stereotyped pursuits and escapes. Although pursuitescape tasks have attracted mathematical interest for 2500 years [43], the effects of reaction times on them have only recently begun to attract attention [23, 59].

Visuomotor tracking tasks are studied by neuroscientists in order to investigate the possibility that the nervous systems employs feedforward, or predictive, control strategies to control complex voluntary movements [33]. However, recent observations suggest that determining a predictive component for control in the presence of noise and delay may be problematic [35, 56, 61]. The goal of the student projects is to determine the extent to which the observed dynamics of virtual stick balancing can be accounted for by a noisy, time-delayed feedback control mechanism. Presumably the observed dynamics which cannot be accounted for in this manner are those under feedforward control.

As a starting point we use random walks to model stochastic control in the presence of time delays [45]. In order to illustrate this approach, consider a 1-D discrete delayed pursuit approximation to the process shown in Figure 5a. Define $X$ as the displacement, $\sqrt{x^{2}+y^{2}}$, from a common reference, where $x, y$ are the spatial coordinates of the mouse (variables related to the mouse are denoted by subscript $m$ ) and the target (related variables denoted 
by subscript $T$ ). If we assume that the movements of the mouse are influenced by the target but not vice versa, then the movements of the target can be modeled as a simple symmetrical random walk whose transition probability is governed by

$$
\begin{aligned}
P_{T}(n, 0) & =\delta_{n, 0} \\
P_{T}(n, t+1) & =\frac{1}{2} P_{T}(n-1, t)+\frac{1}{2} P_{T}(n+1, t)
\end{aligned}
$$

where $P_{T}(n, t)$ is the probability that the target is at position $X_{T}(t)=n$ at time step $t$. The movements of the mouse are given by a delayed random walk whose transition probabilities are biased towards the target. For a delayed random walk the transition probability at the $n$-th step depends on the position of the walker $\tau$ steps before [45] and

$$
\begin{aligned}
P_{m}(n, 0) & =\delta_{n, 0} \\
P_{m}(n, t) & =g(t-1) P_{m}(n-1, t-1)+f(t-1) P_{m}(n+1, t-1)
\end{aligned}
$$

with

$$
\begin{aligned}
& f(t)=\frac{1}{2}\left\{1+\beta\left(X_{m}(t)-X_{T}(t-\tau)\right)\right\} \\
& g(t)=\frac{1}{2}\left\{1-\beta\left(X_{m}(t)-X_{T}(t-\tau)\right)\right\}
\end{aligned}
$$

where $\beta$ is a constant chosen such that $0 \leq \beta\left(X_{m}(t)-X_{T}(t-\tau)\right) \leq 1$. Consequently $f, g$ are quadratic-like functions that describe, respectively, the transition probability for the walker to move in the negative or positive direction [45]. When $\tau=0$, the mouse is biased to step towards the target. However, when $\tau \neq 0$ the situation becomes complicated since, for example, it can happen that the mouse can be transiently biased away from the target. Numerical simulations indicate that the oscillatory nature of the pursuit-escape task observed for human subjects (Figure 5a) can be reproduced in the model (Figure 5b) by increasing $\beta[42]$.

\subsection{The STICKPERSON project}

With advances in technology, biological data sets are rapidly becoming very large. Thus it is essential that students in bio-mathematics be exposed to questions related to how best to represent large data sets, to how best mine large data sets for useful information, and to how best visualize this information. The STICKPERSON project introduces these concepts to students through investigations of the relationship between body posture and balance. The motivation comes from the impending epidemic of falling in aging Western societies. How is the risk of falling for a given subject to be estimated without causing the person to fall? The condition for balance is that the center of gravity of the body falls within its base of support [62]. However, not all balanced postures are equivalent with respect to their ability to enable efficient corrective responses to fall-threatening perturbations [54, 63]. 


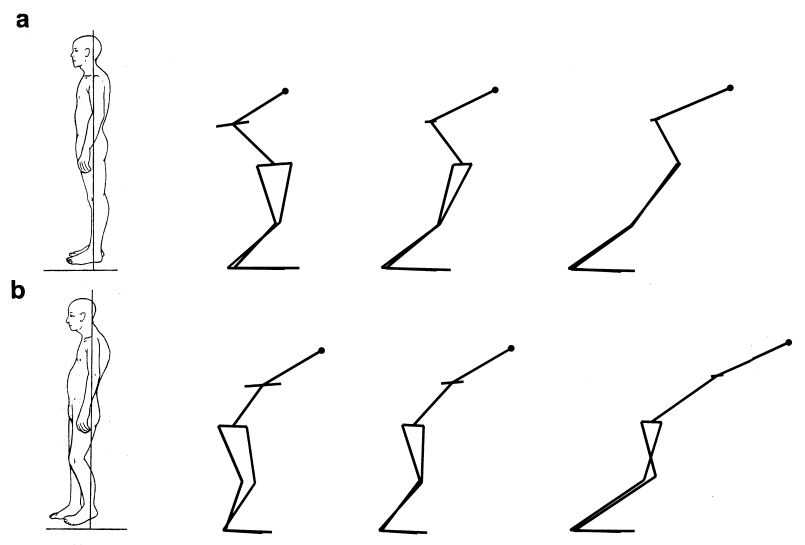

Figure 6: Comparison of the first principal component for postural sway measured during quiet standing using a force platform for a dancer while standing in a) neutral alignment and b) a slouched posture. The stick person representations are given to the right and show three difference multiples of the principal component vector added to the mean position; from left to right, no added contribution, mid-range added contribution, and full range. The range of scaled contributions is arbitrary and is used simply to uncover the nature of the postural information described by the PCA component. Animation of these two STICKPERSONS are provided in the Supplementary materials that accompany the on-line version of this manuscript.

The student team (project onset 2008 involving 8 students to date: one dance-neuroscience dual major, two human biology majors, two neuroscience majors, two oriental medicine, and one mathematics major) asked, Since postural changes occur with aging (compare the postures on the left hand side of Figure 6) are they the cause of increased risk of falling? This hypothesis implies that postural corrections would minimize the risk of falling.

The computational challenge is to understand the responses to perturbations of a spatially distributed and aging musculoskeletal system which evolves in a high-dimensional space. For example, using high speed motion capture techniques to monitor the movements of eleven markers attached to the body (head (1), shoulders (2), hips (2), knees (2), ankles (2) and feet (2)) results in a 33-dimensional space. An important first step is the development of simple, but effective methods for visualizing changes in postural sway based on either linear [47] or nonlinear [15, 26] dimension reduction procedures.

We illustrate our visualization strategy using a linear dimension reduction procedure, namely principal component analysis (PCA). PCA generates a set of orthonormal vectors in $R^{33}$ that acts as a basis for the centered data set, ordered in terms of decreasing variance: e.g., the first basis vector (or principal component vector) spans the dimension along which the motion data has the greatest variance, the second the dimension of next-highest variance, etc. The principal component vectors span the changes in marker positions that occur over the monitoring period. In order to interpret the contribution made by each vector, we added scaled multiples of these vectors to their mean position and graphically represented 
these combinations using a stick person whose coordinates are related to the body markers. Figure 6 shows the effects of a change in posture for a healthy young subject on the first principal component using this scaling visualization approach. The subject was first asked to stand in neutral alignment and then to adopt the slouched posture of an elder subject. By comparing the top and bottom rows of Figure 6 it can be seen that the slouched posture rotates the first principal component vector. A detailed analysis of the changes in the principal components as posture is altered in young healthy and old healthy subjects will be presented elsewhere. The implementation of nonlinear dimension reduction methods, such as diffusion maps [26] and Isomap [15], can capture nonlinear components of the motion and is well within the grasp of undergraduates.

\section{Discussion}

The development of programs for introducing undergraduate students into bio-mathematical research is easiest when modeling efforts are connected to ongoing research at the same institution. In our case we have taken advantage of new technology to create an experimental laboratory for the purpose of bio-mathematical research on balance control and movement. However, there is no reason why programs similar to ours cannot be developed at other institutions by taking advantage of their existing research strengths. The key point is the development of interdisciplinary teams that include both students and faculty. In this way it is possible for young biologists to observe first hand the role of modeling in their research and for budding bio-mathematicians to appreciate how to obtain good data and formulate biological meaningful problems in terms of a mathematical model.

Science education programs are increasingly designed to enable students to learn by doing, rather than by listening. The success rate of these programs becomes particularly high when projects are relevant to student interests and the skills are readily transferable to other problem settings. The use of such experiences allows students to discover for themselves the importance of differential equations for problems that attract their interest: the hope is that once the hook has been set, students will continue to pursue this subject in other areas of science and mathematics. The importance of this hands on introduction to the inter-relationships between science and mathematics cannot be over-emphasized. A motion science program accomplishes these goals in a way that is fun for students and mentors alike!

\section{Acknowledgments}

We thank the faculty and staff who have helped mentor students with interest in motion science including Anna Ahn, Walter Cook, Newt Copp, Vin de Silva, Adam Landsberg, Michele Leblanc, André Longtin, Jim Higdon, Steve Naftilan, Jonathan Snowiss and Francisco Valero-Cuevas. In addition we acknowledge the efforts of students who have participated in the motion science program: Kelsy Avalos-Feehan (CMC), Elizabeth Balch (CMC), Rachel Bellows (PIT), Andrew Blomberg (CMC), Jacob Brooks (PIT), Coury Clemens (CMC), 
Adam C. Coleman (CMC), Jennifer Cummins (CMC), Rachel Dajani (PIT), Emily Deyoe (SCR), Ryan Fraiser (PIT), Cynthia Gama (PIT), Scott M. Garrabrant (PIT), Sydney P. Goings (SCR), Sharday Grant (CMC), Jessica Gunnoe (SCR), Janelle Gyorffy (POM), Joey Haber (PIT), Erin Hanley (SCR), Meredith King (CMC), Joshua Lippai (POM), Y. Leon Liu (HMC), Meela Medhi (POM), Annie L. Nguyentat (CMC), Michael Nguyentat (CMC), David A. Nichols (CMC), Kari C. K. Oki (SCR), Juan Luis Rodriguez (POM), Ferrin A. Ruiz (SCR), Christopher H. Ruth (PIT), Joshua Shapiro (POM), Sky Shanks (PIT), Meredith Strauss (SCR), Molly Tolefson (SCR), Jennifer Townsend (SCR), Ajoy Vase (POM), Atul Vyas (CMC), Lawrence Wang (CMC), Heather Wilson (SCR), Jamie Wong (PIT) and Scott Zimmerman (POM). Finally we acknowledge support from the National Science Foundation (grants NSF-0617072, NSF-1028970, and NSF-0634592), the Ellen Browning Scripps Foundation and the William R. Kenan, Jr. Foundation.

\section{References}

[1] D. Acheson. From Calculus to Chaos: An introduction to dynamics. Oxford University Press, New York (1998).

[2] A. Armenti, Jr., editor. The Physics of Sports. American Institute of Physics, New York (1992).

[3] Y. Asai, Y. Tasaka, K. Nomura, T. Nomura, M. Casidio, P. Morasso A model of postural control in quiet standing: Robust compensation of delay-induced instability using intermittent activation of feedback control. PLoS ONE 4 (2009), e6169.

[4] G. L. Baker, J. A. Blackburn. The pendulum: a case study in physics. Oxford University Press, New York, 2005.

[5] H. C. Berg. Random walks in biology. Princeton University Press, New Jersey (1993).

[6] A. Bottaro, Y. Yasutake, T. Nomura, M. Casidio, P. Morasso. Bounded stability of the quite standing position: An intermittent control model. Human Movement Science 27 (2008), 473-495.

[7] R. Bormann, J. L. Cabrera, J. G. Milton, C. W. Eurich. Visuomotor tracking on a computer screen: An experimental paradigm to study dynamics of motor control. Neurocomputing 58-60 (2004), 517-523.

[8] J. Boulet, R. Balasubramiam, A. Daffertshofer, A. Longtin. Stochastic two-delay differential model of delayed visual feedback effects on postural dynamics. Phil. Trans. Roy. Soc. A 368 (2010): 423-438.

[9] J. L. Cabrera, J. G. Milton. On-off intermittency in a human balancing task. Phys. Rev. Lett. 89 (2002), 158702. 
[10] J. L. Cabrera, J. G. Milton. Human stick balancing: Tuning Lévy flights to improve balance control. CHAOS 14 (2004): 691.

[11] J. L. Cabrera, R. Bormann, C. Eurich, T. Ohira, J. Milton. State-dependent noise and human balance control. Fluct. Noise Lett. 4 (2004), L107-L118.

[12] S. A. Campbell, S. Crawford, K. Morris. Friction and the inverted stabilization problem. J. Dyn. Syst. Meas. Control. 130 (2008), 054502.

[13] J. J. Chiel, R. D. Beer. The brain has a body: adaptive behavior emerges from interactions of nervous system, body and environment. TINS 20 (1997), 553-557.

[14] T. Cluff, R. Balasubramaniam R. Motor learning characterized by changing Lévy distributions. PLoS One 4 (2009): e5998.

[15] V. de Silva, J. B. Tenenbaum, J. C. Langford. A global geometric framework for nonlinear dimensionality reduction. Science 290 (2000), 2319-2323.

[16] T. M. H. Dijkstra, H. Katsumata, D. Sternad. The dialogue between data and model: passive stability and relaxation behavior in a ball bouncing task. Nonlinear Studies 11 (2004), 319-344.

[17] B. Ermentrout. Simulating, Analyzing, and Animating Dynamical Systems. SIAM, Philadelphia (2002).

[18] C. W. Eurich, J. G. Milton JG. Noise-induced transitions in human postural sway. Phys. Rev. E 54 (1996): 6681-6684.

[19] C. W. Eurich, K. Pawelzik. Optimal control yields power laws. In Artificial Neural Networks: Formal Models and Their Applications, Springer Lecture Notes in Computer Science Vol. 3697, edited by W. Duch, J. Kacprzyk, E. Oja and S. Zadronzny (SpringerVerlag, Berlin, 2005), pp. 365-370.

[20] P. Foo, J. A. S. Kelso, G. D. de Guzman. Functional stabilization of fixed points: Human pole balancing using time to balance information. J. Exp. Psychol. Hum. Percept. Perform. 26 (2000), 1281-1297.

[21] J. Guckenheimer. A robust hybrid stabilization strategy for equilibria. IEEE Trans. Automatic Control 40 (1995), 321-326.

[22] T. Insperger. Stick balancing with reflex delay in case of parametric forcing. Commun. Nonlinear Sci. Numer. Simulat. 16 (2011), 2160-2168.

[23] A. Kamimura, T. Ohira. Group chase and escape. New J. Physics 12 (2010), 053013.

[24] T. A. Kuiken, L. A. Miller, R. D. Lipschutz, B. A. Lock, K. Stubblefield, P. D. Marasso, P. Zhou, G. A. Dumanian. Targeted reinnervation for enhanced prosthetic arm function in a woman with a proximal amputation: a case study. Lancet 369 (2007), 371-380. 
[25] A. D. Kuo. The six determinants of gait and the inverted pendulum analogy: A dynamic walking perspective. Hum. Mov. Sci. 26 (2007), 617-656.

[26] S. S. Lafon. Diffusion Maps and Geometric Harmonics. PhD thesis, Yale University, 2004.

[27] M. Landry, S. A. Campbell, K. Morris, C. O. Aguilar. Dynamics of an inverted pendulum with delayed feedback control. SIAM J. Appl. Dyn. Sys. 4 (2005), 333-351.

[28] D. B. Lockhart, L. H. Ting. Optimal sensorimotor transformations for balance. Nat. Neurosci. 10 (2007), 1329-1336.

[29] I. D. Loram, M. Lackie. Human balancing of an inverted pendulum: position control by small, ballistic-like, throw and catch movements. J. Physiol. (London) 540 (2002), 1111-1124.

[30] I. D. Loram, C. N. Maganaris, M. Lakie. Human postural sway results from frequent, ballistic bias impulses by soleus and gastrocnemius. J. Physiol. (London) 564 (2005), 295-311.

[31] J. Maynard Smith. Mathematical Ideas in Biology. Cambridge University Press, New York (1968).

[32] T. A. McMahon. Muscles, Reflexes and Locomotion. Princeton University Press, New Jersey (1984).

[33] B. Mehta, S. Schaal. Forwards models in visuomotor control. J. Neurophysiol. 88 (2002), 942-953.

[34] J. G. Milton, S. S. Small, A. Solodkin. On the road to automatic: Dynamic aspects in the development of expertise. J. Clin. Neurophysiol. 21 (2004), 134-143.

[35] J. G. Milton, J. L. Cabrera, T. Ohira. Unstable dynamical systems: Delays, noise and control. Europhys. Lett. 83 (2008), 48001.

[36] J. G. Milton, T. Ohira, J. L. Cabrera, R. M. Fraiser, J. B. Gyorffy, F. K. Ruiz, M. A. Strauss, E. C. Balch, P. J. Marin, J. L. Alexander. Balancing with vibration: A prelude for "drift and act" balance control. PLOS One 4 (2009), e7427.

[37] J. Milton, J. L. Cabrera, T. Ohira, S. Tajima, Y. Tonosaki, C. W. Eurich, S. A. Campbell. The time-delayed inverted pendulum: Implications for human balance control. Chaos 19 (2009), 026110.

[38] J. Milton, J. L. Townsend, M. A. King, T. Ohira. Balancing with positive feedback: the case for discontinuous control. Phil. Trans. Roy. Soc. A 367 (2009), 1181-1193. 
[39] J. Milton, J. Gyorffy, J. L. Cabrera, T. Ohira. Amplitude control of human postural sway using Achilles tendon vibration. 16th US National Congress of Theoretical and Applied Mechanics (2010). State College, PA (USNCTAM2010-791).

[40] J. G. Milton, A. E. Radunskaya, A. H. Lee, L. G. de Pillis, D. F. Bartlett. Team research at the biology-mathematics interface: Project management perspectives. CBELife Sciences Education 9 (2010), 316-322.

[41] J. Milton, P. Naik, C. Chan, S. A. Campbell. Indecision is neural decision making models. Math. Model. Nat. Phenom. 5 (2010), 125-145.

[42] J. Milton, J. Lippai, R. Bellows, A. Blomberg, A. Kamimura, T. Ohira. Visuomotor tracking tasks with delayed pursuit and escape. 8th International Conference on Multibody Systems, Nonlinear Dynamics and Control (2011). Washington, D. C. (DETC201147312).

[43] P. J. Nahin PJ. Chases and escapes: The mathematics of pursuit and evasion. Princeton University Press, Princeton, New Jersey (2007).

[44] http://www.gnu.org/software/octave/

[45] T. Ohira, J. Milton. Delayed random walks: Investigating the interplay between noise and delays. In: Delay Differential Equations: Recent Advances and New Directions, edited by B. Balachandran, T. Kalmár-Nagy and D. E. Gilman, Springer-Verlag, New York, pp. 305-335 (2009).

[46] F. Patzelt, M. Riegel, U. Ernst, K. Pawelzik. Self-organized critical noise amplification in human closed loop control. Front. Comp. Neurosci. 1 (2007), Article 4, 1-9.

[47] I. J. Pinter, R. von Swigchem, A. J. Knoek van Soet, L. A. Rozendaal. The dynamics of postural sway cannot be captured using a one-segment inverted pendulum model: A PCA on segment rotations during unperturbed stance. J. Neurophysiol. 100 (2008), 3197-3208.

[48] A. B. Pippard. The inverted pendulum. Eur. J. Physics 8 (1987), 203-206.

[49] http://pydelay.sourceforge.net/

[50] https://code.astraw.com/projects/PyUniversalLibrary/

[51] http://www.sagemath.org/

[52] http://www.scipy.org/

[53] S. H. Scott. Optimal feedback control and the neural basis of volitional motor control. Nature Rev, Neurosci. 5 (2004), 534-546.

[54] J. R. Stirling, M. S. Zakynthinaki. Stability and the maintenance of balance following a perturbation from quiet stance. Chaos 14 (2004), 96-105. 
[55] G. Stepan. Delay effects in the human sensory system during balancing. Phil. Trans. Roy. Soc. A 367 (2009), 1195-1212.

[56] N. Stepp. Anticipation in feedback-delayed manual tracking tracking of a chaotic oscillator. Exp. Brain Res. 198 (2009), 521-525.

[57] A. Straw. An open-source library for realtime visual stimulus generation. Frontiers Neuroinformatics 11 (2008): doi: 10.3389.neuro.11.004:2008.

[58] A. D. Straw, M. H. Dickinson. Motmot, an open-source toolkit for realtime video acquisition and analysis. Source Code for Biology and Medicine (2010). doi: 10.1186/17510473-4-5.

[59] T. Vicsek. Closing in on evaders. Nature 466 (2010), 43-44.

[60] S. Vogel. Comparative Biomechanics: Life's physical world. Princeton University Press, New Jersey (2003).

[61] H. U. Voss. Anticipating chaotic synchronization. Phys. Rev. E 61 (2000), 5115-5119.

[62] D. A. Winter, A. e. Patla, F. Prince, M. Ishac, K. Gielo-Perczak. Stiffness control in quiet standing. J. Neurophysiol. 80 (1998), 1211-1221.

[63] M. S. Zakynthinaki, J. M. Madera Milla, A. López de Durana, C. A. Cordent Martinez, G. Rodriguez Romo, M. Stillero Quintana, J. Samperdo Molinuevo. Rotated balance in humans due to repetitive rotational movement. Chaos 20 (2010), 013118.

[64] V. Zatsiorsky. Biomechanics in Sports: Performance enhancement and injury prevention. Blackwell Science, Malden, MA (2000). 